\title{
Promoção da saúde mental e enfrentamento ao bullying no ambiente escolar
}

\author{
Promoting mental health and tackling bullying in the school environment \\ Promover la salud mental y abordar el acoso escolar en el entorno escolar
}

Recebido: 06/04/2021 | Revisado: 15/04/2021 | Aceito: 27/04/2021 | Publicado: 11/05/2021

\begin{tabular}{|c|}
\hline $\begin{array}{r}\text { Cristefânia Meirú de Lima } \\
\text { ORCID: https://orcid.org/0000-0001-8919-4988 } \\
\text { Universidade da Integração Internacional da Lusofonia Afro-Brasileira, Brasil }\end{array}$ \\
\hline $\begin{array}{l}\text { E-mail: crismeiru@gmall.com } \\
\text { Antonio Rubens Alves da Silva }\end{array}$ \\
\hline ORCID: https://orcid.org/0000-0001-7328-3388 \\
\hline Universidade da Integração Internacional da Lusofonia Afro-Brasileira, Brasil \\
\hline E-mail: rubens@aluno.unilab.edu.br \\
\hline Andreia de Melo Mendonça \\
\hline ORCID: https://orcid.org/0000-0003-1778-6970 \\
\hline Universidade da Integração Internacional da Lusofonia Afro-Brasileira, Brasil \\
\hline E-mail: andreia.melom@gmail.com \\
\hline Nathanael de Souza Maciel \\
\hline ORCID: https://o \\
\hline gração Internacional da Lusofonia Afro-Brasileira, Brasil \\
\hline E-mail: nathanael.souza.inf@gmail.com \\
\hline Naara Ingrid da Silva Sales \\
\hline ORCID: https://orcid.org/0000-0003-2619-5558 \\
\hline Universidade da Integração Internacional da Lusofonia Afro-Brasileira, Brasil \\
\hline E-mail: naarayngridjesus@gmail.com \\
\hline Eysler Gonçalves Maia Brasil \\
\hline ORCID: https://orcid.org/0000-0002-4126-2256 \\
\hline $\begin{array}{r}\text { Universidade da Integração Internacional da Lusofonia Afro-Brasileira, Brasil } \\
\text { E-mail: eyslerbrasil@ unilab.edu.br }\end{array}$ \\
\hline
\end{tabular}

\section{Resumo}

Objetivo: Relatar a experiência vivenciada por acadêmicos de enfermagem durante a realização de ações de promoção da saúde mental, focando o bullying com crianças escolares. Metodologia: Trata-se de um estudo descritivo do tipo relato de experiência, realizou-se um encontro no período da manhã com crianças entre 9 a 11 anos em uma escola de ensino fundamental no interior do Ceará. Utilizou-se a observação e a descrição da atividade, em que os dados obtidos foram analisados baseados na literatura atual sobre a temática. A atividade foi realizada seguindo quatro etapas: (1) encenação de um livro infantil; (2) dinâmica quebra-gelo intitulada "diferenças"; (3) exploração da temática bullying; e (4) arteterapia. Resultados: Percebeu-se que as ações de promoção da saúde mental no ambiente escolar, assim como as temáticas abordadas no enfrentamento ao bullying são importantes e as estratégias utilizadas pelos discentes atingiram seus objetivos, evidenciado pelo interesse, pela escuta e pelo diálogo no grupo e a participação ativa das crianças durante todas as etapas executadas. Destaca-se ainda que o uso da arteterapia foi fundamental para a fixação do conteúdo abordado. Conclusão: A atividade realizada propiciou familiaridade com a temática e o uso de metodologias ativas, contribuindo para a formação dos discentes, ampliando o conhecimento teórico e subjetivo sobre o assunto abordado, bem como a promoção da saúde mental das crianças no ambiente escolar.

Palavras-chave: Bullying; Criança; Enfermagem; Saúde mental.

\begin{abstract}
Objective: To report the experience lived by nursing students during the execution of actions to promote mental health, focusing on bullying with school children. Methodology: This is a descriptive study of the type of experience report, a meeting was held in the morning with children between 9 and 11 years old in an elementary school in the interior of Ceará. Observation and description of the activity were used, in which the data obtained were analyzed based on the current literature on the subject. The activity was carried out following four steps: (1) staging a children's book; (2) icebreaker dynamics entitled "differences"; (3) exploration of the theme of bullying; and (4) art therapy. Results: It was noticed that the actions to promote mental health in the school environment, as well as the themes addressed in the fight against bullying are important and the strategies used by the students reached their goals, evidenced by the interest, listening and dialogue in the group and participation active participation of children during all stages performed. It is also noteworthy that the use of art therapy was fundamental for fixing the content covered. Conclusion: The activity carried out provided familiarity with the theme and the use of active methodologies,
\end{abstract}


contributing to the training of students, expanding theoretical and subjective knowledge about the subject addressed, as well as promoting the mental health of children in the school environment.

Keywords: Bullying; Child; Nursing; Mental health.

\section{Resumen}

Objetivo: Informar la experiencia vivida por los estudiantes de enfermería durante la ejecución de acciones de promoción de la salud mental, con foco en el acoso escolar con los escolares. Metodología: Se trata de un estudio descriptivo del tipo de relato de experiencia, se realizó un encuentro por la mañana con niños de entre 9 y 11 años en una escuela primaria del interior de Ceará. Se utilizó la observación y descripción de la actividad, en la que se analizaron los datos obtenidos en base a la literatura actual sobre el tema. La actividad se llevó a cabo siguiendo cuatro pasos: (1) montaje de un libro infantil; (2) dinámica de rompehielos titulada "diferencias"; (3) exploración del tema del acoso escolar; y (4) arteterapia. Resultados: Se notó que las acciones para promover la salud mental en el ámbito escolar, así como los temas abordados en la lucha contra el acoso escolar son importantes y las estrategias utilizadas por los estudiantes alcanzaron sus metas, evidenciado por el interés, la escucha y el diálogo en el grupo. y participación participación activa de los niños durante todas las etapas realizadas. También es de destacar que el uso de la arteterapia fue fundamental para fijar el contenido cubierto. Conclusión: La actividad realizada brindó familiaridad con el tema y el uso de metodologías activas, contribuyendo a la formación de los estudiantes, ampliando los conocimientos teóricos y subjetivos sobre el tema abordado, además de promover la salud mental de los niños en el ámbito escolar.

Palabras clave: Acoso Escolar; Niño; Enfermería; Salud mental.

\section{Introdução}

O processo de escolarização exerce uma atribuição essencial na construção do indivíduo. As repercussões desse período não podem ser observadas sem considerar os contextos de inserção do sujeito, especialmente as diferentes práticas culturais. Esse emaranhado de vivências resulta em novas construções afetivas e também pode gerar adversidades. Nesse âmbito, o tema bullying apresenta-se como importante fenômeno a ser compreendido e contextualizado (Toro, Neves \& Rezende, 2010). Refere-se bullying no espaço escolar na ocasião em que a criança é exposta a atitudes e práticas propositalmente ultrajantes por outras pessoas, de forma recorrente e prolongada. Esses atos violentos objetivam deteriorar, lesar e abalar a criança e instituir um relacionamento onde dois atores emergem: o dominante e o dominador. Frequentemente, o provocador é um integrante do ambiente escolar ou um agrupamento de condiscípulos (Souza, 2019).

Quanto à característica e aos conteúdos, o bullying pode ser tradicional, que compreende comportamentos agressivos de natureza física (socos, pontapés, empurrões e outros), verbal (fofocas, xingamentos, apelidos, entre outros) e psicológico (ameaças, insultos e chantagens) (Silva, Silva, Pereira, Oliveira \& Medeiros, 2014). Essas situações desencadeiam um efeito prejudicial a respeito da possibilidade de adquirir conhecimentos, assim como acerca da autoestima da criança, e, por conseguinte, um comprometimento de sua saúde mental (Souza, 2019). Vítimas de bullying podem manifestar maior risco de ideação e comportamentos suicidas. Neste sentido, os impactos decorrentes do bullying na saúde mental dos indivíduos tornase um sério problema de saúde pública, reivindicando, de dessemelhantes segmentos sociais, estratégias que possa minimizálos (Costa \& Miranda, 2020).

Diante desse cenário, as ações de promoção da saúde mental nas escolas tornam-se imprescindíveis, em que focam na identificação das necessidades das crianças e dos adolescentes, baseadas no diálogo, prevenindo assim o adoecimento psíquico, a fim de obter-se melhor qualidade de vida (O'Reilly, Svirydzenka, Adams \& Dogra, 2018). Tal fato encontra-se de acordo com o que a Organização Mundial da Saúde (OMS) preconiza ao definir a promoção da saúde mental como ações que criem condições e ambientes de vida, os quais devem apoiar a saúde mental e permitir que os indivíduos passem a adotar e manter estilos de vida saudáveis (Organização Mundial da Saúde, 2016). Salienta-se que as esferas da saúde e da educação carecem conceber um enfoque fundamentado no cuidado e no âmbito da promoção da saúde, a nível individual e coletivo, com recursos interdisciplinares e intersetoriais (Mello, Silva, Oliveira, Prado, Malta \& Silva, 2017). Nessa perspectiva, se insere a Atenção Primária à Saúde (APS), reconhecendo as necessidades biopsicossociais, individuais e coletivas, ampliando a emancipação e 
empoderamento dos indivíduos, por meio de ações de promoção da saúde e prevenção de doenças e agravos (Brasil, 2017).

Os profissionais da APS, sobretudo enfermeiros, podem intervir neste cenário, com o intuito de mapear situações que podem estar ou não associadas à manifestação do bullying. Estes profissionais oportunizam, ainda, a organização de projetos para a redução de comportamentos agressivos, a resolução de conflitos ou o desenvolvimento de habilidades sociais, além do trabalho junto às famílias, o que ganha força com as propostas de cultura de não violência e de políticas na área (Silva, Silva, Pereira, Oliveira \& Medeiros, M., 2014). Dentre as competências de enfermeiros que atuam na APS, evidencia-se a assistência à saúde aos indivíduos e famílias, podendo usufruir, quando indicado ou necessário, de espaços comunitários, como escolas, e trabalhar a intersetorialidade (Brasil, 2017). Desse modo, na operacionalização do Programa Saúde na Escola, os enfermeiros podem atuar diretamente nas instituições escolares com vistas à realização de diagnósticos dos problemas, enfocando a problemática do bullying, bem como na identificação e no planejamento de intervenções específicas para a comunidade educacional, vítimas, agressores, famílias e comunidade (Silva, Silva, Pereira, Oliveira \& Medeiros, 2014).

Evidencia-se uma falta de estudos de ações de promoção da saúde e uma lacuna na pesquisa sobre prevenção de bullying, práticas restaurativas e os impactos destas ações. Além de preencher essa lacuna de pesquisa, há uma necessidade de ações e estudos que investiguem e descrevam as experiências e percepções dos sujeitos envolvidos no bullying e avaliem programas de intervenção intersetorial que abrangem escolas, serviços de saúde e outros setores da comunidade (Pigozi, 2018). Nesse panorama, objetivou-se relatar a experiência vivenciada por acadêmicos de enfermagem durante a realização de ações de promoção da saúde e enfrentamento ao bullying com crianças escolares.

\section{Metodologia}

Trata-se de um estudo descritivo, do tipo relato de experiência vivenciado pelos discentes do $6^{\circ}$ semestre do curso de graduação em enfermagem, de uma universidade federal e internacional localizada no interior do Estado do Ceará. A ação foi realizada no período da manhã em uma sala de aula contendo vinte alunos de uma escola de ensino fundamental, também localizada no Ceará, como atividade prática proposta pela disciplina "Processo de Cuidar na Saúde Mental", a qual foi acompanhada pela professora e com a anuência da gestão da escola.

A técnica de grupo operativo é baseada na formação de grupos de trabalho, sendo o objetivo principal a promoção de um processo de aprendizagem para os sujeitos que participam. Dessa forma, aprender coletivamente trata-se de uma rede de interações em que é realizada uma leitura da realidade, desde que seja de forma crítica, bem como incentivada atitude investigadora, abertura para dúvidas e novas inquietações. Nesse sentido, as interações fazem com que o sujeito possa referenciar-se no outro, encontrar-se com o outro, diferenciar-se do outro, opor-se a ele e, assim, transformar e ser transformado por este. Com isso, a técnica do grupo operativo abrange etapas fundamentais que são a tarefa explícita (aprendizagem, diagnóstico ou tratamento), a tarefa implícita (o modo como cada integrante vivencia o grupo) e o enquadre ou elementos fixos (o tempo, a duração, a frequência, a função do coordenador e do observador) (Pichon-Rivière, 1988; Bastos, 2010).

O grupo operativo foi realizado com crianças, com faixa etária entre 9 a 11 anos. A ação foi dividida em quatro momentos: (1) encenação do livro "O Ernesto", por meio de uma dramatização da história infantil; (2) dinâmica quebra-gelo intitulada "diferenças"; (3) brainstorming (tempestade de ideias) para explicação sobre bullying; (4) arteterapia.

No primeiro momento, realizou-se uma encenação do livro "Ernesto", de Blandina Franco e José Carlos Lollo, que conta a história de um menino descrito como diferente e que, consequentemente, algumas pessoas achavam-no esquisito, não muito simpático, e até mesmo que ele não sabia se vestir. No segundo momento, empregou-se uma dinâmica quebra-gelo intitulada "Diferenças", onde as crianças tinham que desenhar individualmente de acordo com as coordenadas dadas pelos discentes, como: "desenhe um rosto", "um braço", "uma perna" de modo que, ao fim dos comandos, tinha-se um corpo 
completo desenhado por cada criança. No terceiro momento, abordou-se a temática bullying, de forma interativa, apresentando conceitos, tipos, implicações e impactos, bem como a ênfase sobre buscar ajuda. O último momento foi destinado ao uso da arteterapia para promover a saúde mental das crianças, em que tinham que recontar a história encenada ao criar um novo desfecho de acordo com os conteúdos abordados. Por fim todos ficaram em volta de um círculo e de mãos dadas, onde cada um deveria fazer um elogio à pessoa que estava do seu lado e, após esse momento, todos se abraçaram coletivamente.

Ressalta-se que por se tratar de um relato de experiência, o estudo não passou pelo comitê de ética. No entanto, foi solicitada previamente a autorização da direção da escola para a realização da ação. Os dados dos participantes não foram divulgados, respeitando as normas preconizadas pela Resolução 466/2012 do Conselho Nacional de Ética em Pesquisa (Brasil, 2012).

\section{Resultados e Discussão}

Percebeu-se que as crianças foram receptivas e demonstraram interesse na temática, na apresentação dos membros da equipe e quais eram os objetivos do grupo operativo, além disso, elas também se apresentaram. No primeiro momento da encenação, as crianças ficaram surpresas e empolgadas ao verem os acadêmicos caracterizados, tal fato colaborou para chamar a atenção delas e abordar uma temática prevalente na escola, o bullying, onde também foi uma necessidade identificada pela gestão da escola. Assim, quando foi discutida a temática bullying, observou-se que a maioria dos escolares tinham conhecimentos prévios acerca do assunto, foram interativos, bem como relaram que já tinham passado por tal situação, no entanto, apenas sete alunos quiseram se expressar.

No que diz respeito ao planejamento da ação realizou-se pesquisas sobre como abordar as crianças, sendo selecionadas formas de metodologias ativas. Observa-se que as novas tendências pedagógicas, como as metodologias ativas, costumam ser estratégias onde o aluno aparece como protagonista principal. Logo, quem medeia nesses contextos apresentamse como facilitadores das experiências vivenciadas no processo de aprendizagem, fazendo com que as metodologias empregadas oportunizem o resgate do contexto vivenciado e das características individuais (Alves, Marx, Bezerra \& Landim, 2017).

Sabe-se que a incidência de bullying costuma ser elevada nas séries iniciais, além disso, as crianças apresentam um intenso desenvolvimento nesse período e as ações executadas interferem diretamente em formas de pensar e/ou agir. Diante desse contexto, pode-se dizer que as escolas devem adotar um posicionamento positivo em relação à promoção da saúde mental, a fim de combater a alta prevalência de transtornos mentais (O'Reilly, Svirydzenka, Adams \& Dogra, 2018). No entanto, ao considerar a escola como uma organização de grande dimensão, pode-se observar um lugar de vulnerabilidade para os que estão inseridos nesse espaço, pois podem ocorrer diversos tipos de violência e de agressividade, desde as expressadas fisicamente até as formas psicológicas (Matos, Martins, Jesus \& Viseu, 2015).

Acerca das atividades que exigiam desenhos dos escolares, observou-se que foi executada de forma prazerosa, mas o conteúdo dos desenhos foi fortemente influenciado pelo que foi discutido em sala, visto que eles mostraram compreensão sobre a abordagem e mudança de conceitos pré-formulados. Notou-se que elas ficaram bem reflexivas ao relatarem que os desenhos, resultantes da dinâmica quebra-gelo intitulada "diferenças" realizada no segundo momento do grupo operativo, implicavam diferentes formas de imaginar um mesmo comando, já que não tinha a forma correta para o desenho, sendo importante respeitar as diferenças do outro, incentivando a criatividade e a imaginação.

Diante disso, nota-se que a crescente utilização de arteterapia prova ser eficaz para melhorar os aspectos emocionais das pessoas, principalmente de crianças, pois a técnica desperta a consciência sobre a realidade imediatamente e captura através de vários meios (Sansalonis, 2016). Assim, ao adotar esse tipo de linguagem não verbal permite-se que os indivíduos expressem suas emoções e sentimentos, tal como observado na terapia, em que as expressões artísticas são incentivadas para 
ajudar o paciente em alguns de seus problemas. Logo, quando empregou-se a arteterapia ela foi executado de forma agradável, pois os escolares ficaram bem concentrados e ao final da atividade os desenhos foram explicados e colados no mural da sala de aula. Tal momento foi imprescindível para fixação do conteúdo aprendido, pois foram feitas comparações com o que as crianças disseram inicialmente e o que achavam após as experiências vivenciadas.

Além disso, no período entre o nascimento e os dez anos de idade, o cérebro da criança está em pleno desenvolvimento e apresenta maiores e melhores condições para o aprendizado (Ilari, 2006; Said \& Abramides, 2020). Assim, o desenvolvimento infantil costuma ser marcado por intensas mudanças que vão se consolidando de acordo com as relações sociais estabelecidas. Logo, considerando o bullying como uma prática recorrente no ambiente escolar e responsável por uma série de consequências que podem interferir nessas relações (Zequinão, Medeiros, Silva, Pereira \& Cardoso, 2020), torna-se fundamental realizar ações que promovam a saúde mental das crianças no contexto escolar.

A expectativa criada inicialmente era que a maioria dos alunos participassem e interagissem com os acadêmicos que iriam direcionar o momento, a fim de formar-se um vínculo com a atenção direcionada para o assunto e fixação de conteúdo, como também a promoção da saúde mental. Porém, existia-se receio devido a pouca experiência em se trabalhar com um público infantil. Felizmente, considerando-se as observações do grupo e comentários das crianças, conseguiu-se constatar que as crianças mostraram interesse pelo assunto, tal fato demonstrado pela interação e demonstração em todas as etapas. Por fim, a atividade possibilitou aos acadêmicos uma experiência enriquecedora.

Ademais, é importante destacar que o grupo operativo proporciona um momento de troca de conhecimentos, de interação dos próprios membros uns com os outros, para que desta forma identifiquem quem mais precisa de ajuda, contando com o apoio dos profissionais da educação e da saúde. Além disso, proporciona uma confiança maior nos profissionais, para que desta forma, possam desenvolver uma escuta ativa e que tenham uma relação melhor com as crianças. Portanto, as atividades grupais são de essencial importância enquanto práticas educativas para o desenvolvimento humano, ajudando as pessoas a relacionar-se melhor consigo mesma, com o outro e com o mundo (Mazzuchello, Ceretta, Schwalm, Dagostim \& Soratto, 2014).

Desta forma, com o trabalho fica perceptível como é importante que o profissional que vai atuar com este público tenha conhecimento na área da educação e da saúde mental, para contribuir de forma significativa na identificação das necessidades desta clientela, dos encaminhamentos quando necessário, e que no campo da profissão ajam de forma ativa com intervenções que contribuam com a qualidade de vida destas crianças. Ou seja, os profissionais de enfermagem que atuam na atenção básica e no Projeto Saúde na Escola devem fazer uso de grupos operativos, a fim de realizar ações de promoção da saúde mental, sendo tais fatos atribuições de seu campo de atuação.

Tais fatos são reforçados pelos trabalhos com essa modalidade grupal sendo utilizados por profissionais de saúde, tendo em vista a capacidade operativa e educativa do grupo em promover o acolhimento, ressignificação das experiências e a aprendizagem construída com a interação dos participantes (Oliveira, Rena, Mendonça, Pereira, Jesus \& Merighi, 2016). Nessa perspectiva, pelo fato da enfermagem ser a categoria profissional que comumente realiza atividades grupais nos diversos cenários de assistência em saúde, o enfermeiro se destaca como um dos principais atores que integra a equipe interdisciplinar na abordagem às crianças (Pinheiro, Araújo, Rolim, Oliveira \& Alencar, 2019).

\section{Conclusão}

Ao finalizar a atividade percebeu-se que apesar da familiaridade com o tema, nota-se que ele continua presente na vivência entre os escolares em estudo. Tal fato reforça a abordagem da temática dentro do ambiente escolar, fazendo-se uso das diversas metodologias ativas, tais como as utilizada nesta ação, no enfrentamento ao bullying e a promoção da saúde mental dos escolares. 
Entende-se que a atividade teve uma importante contribuição para a formação dos discentes ao tocante do desenvolvimento de habilidades técnicas e comunicativas, criação de vínculos, trabalho em equipe. Dessa maneira, a realização da ação educativa e de promoção da saúde mental forneceu subsídios para a ampliação do conhecimento teórico e subjetivo sobre a temática abordada.

\section{Referências}

Alves, M. N. T., Marx, M., Bezerra, M. M. M., \& Landim, J. M. M. (2017). Metodologias Pedagógicas Ativas na Educação em Saúde. Revista Multidisciplinar e de Psicologia, 10(33), 112-125. https://doi.org/10.14295/idonline.v10i33.659

Bastos, A. B. B. I. (2010). A técnica de grupos-operativos à luz de Pichon-Rivière e Henri Wallon. Psicologo informacao, 14(14), 160-169. http://pepsic.bvsalud.org/scielo.php?script=sci_arttext\&pid=S1415-88092010000100010\&lng=pt\&tlng=pt.

Brasil. (2012). Resolução 466 de 12 de dezembro de 2012: que trata de pesquisas com seres humanos e atualiza a resolução 196. https://conselho.saude.gov.br/resolucoes/2012/Reso 466.pdf

Brasil. (2017). Portaria N $\mathrm{N}^{\circ}$ 2.436, de 21 de setembro 2017. Aprova a Política Nacional de Atenção Básica, estabelecendo a revisão de diretrizes para a organização da Atenção Básica, no âmbito do Sistema Único de Saúde (SUS). http://bvsms.saude.gov.br/bvs/saudelegis/gm/2017/prt2436_22_09_2017.html

Costa, K. M. R., \& Miranda, C. E. S. (2020). Associação entre bullying escolar e suicídio: uma revisão integrativa da literatura. Cad Bras Saúde Ment J Ment Health, 12 (31), 312-327. https://periodicos.ufsc.br/index.php/cbsm/article/view/69815

Ilari, B. (2006). Música, comportamento social e relações interpessoais. Psicol Estud, 11 (1), 191-198. https://doi.org/10.1590/S1413-73722006000100022

Matos, F., Martins, H., Jesus, S. N., \& Viseu, J. (2015). Prevenção da violência através da resiliência dos alunos. Psic., Saúde \& Doenças, 16 (1), $35-43$. http://dx.doi.org/10.15309/15psd160105

Mazzuchello, F. R., Ceretta, L. B., Schwalm, M. T., Dagostim, V. S., \& Soratto, M. T. (2014). A atuação dos enfermeiros nos grupos operativos terapêuticos na estratégia saúde da família. O Mundo da Saúde, 38 (4), 462-472. http://www.saocamilo-sp.br/pdf/mundo_saude/155566/A11.pdf

Mello, F. C. M., Silva, J. L., Oliveira, W. A., Prado, R. R., Malta, D. C., \& Silva, M. A. I. (2017). A prática de bullying entre escolares brasileiros e fatores associados, Pesquisa Nacional de Saúde do Escolar 2015. Ciênc Amp Saúde Coletiva, 22 (9), 2939-2948. https://doi.org/10.1590/141381232017229.12762017

O'Reilly, M., Svirydzenka, N., Adams, S., \& Dogra, N. (2018). Revisão das intervenções de promoção da saúde mental nas escolas. Soc Psychiatry Psychiatr Epidemiol, 53, 647-662. https://doi.org/10.1007/s00127-018-1530-1

Oliveira, D. M., Rena, P. B. O., Mendonça, E. T., Pereira, E. T., Jesus, M. C. P., \& Merighi, M. A. B. (2016). O grupo operativo como instrumento de aprendizagem do cuidado por mães de filhos com deficiência. Escola Anna Nery, 20(3), e20160077. https://doi.org/10.5935/1414-8145.20160077

Organização Mundial da Saúde. (2016). Saúde mental: fortalecendo nossa resposta. http://www.who.int/mediacentre/factsheets/fs220/en/

Pichon-Rivière, E. (1988). Teoria do vínculo. São Paulo: Martins Fontes.

Pigozi, P. L. (2018). A produção subjetiva do cuidado: uma cartografia de bullying escolar. Physis Rev Saúde Coletiva, 28 (3), e280312. https://doi.org/10.1590/s0103-73312018280312

Pinheiro, C. W., Araújo, M. A. M., Rolim, K. M. C., Oliveira, C. M., \& Alencar, A. B. (2019). Teoria das relações interpessoais: reflexões acerca da função terapêutica do enfermeiro em saúde mental. Rev. Enfermagem em Foco, 10(3), 64-69. http://revista.cofen.gov.br/index.php/enfermagem/article/view/2291/580

Said, P. M., \& Abramides, D. V. M. (2020). Efeito da educação musical na promoção do desempenho escolar em crianças. CoDAS, 32 (1), e20180144. https://doi.org/10.1590/2317-1782/20192018144

Sansalonis, P. J. R. (2016). Arte-terapia e Síndrome de Asperger: porquê e para quê. Sophia, 12 (2), 187-194. https://doi.org/10.18634/sophiaj.12v.2i.322

Silva, M. A. I., Silva, J. L., Pereira, B. O., Oliveira, W. A.., \& Medeiros, M. (2014). O olhar de professors sobre o bullying e implicações para a atuação da enfermagem. Rev Esc Enferm USP, 48 (4), 723-730. https://doi.org/10.1590/S0080-623420140000400021

Souza, L. (2019). Quando o bullying na escola afeta a vida adulta. Rev Psicopedagogia, $36 \quad$ (110),153-162. https://cdn.publisher.gn1.link/revistapsicopedagogia.com.br/pdf/v36n110a04.pdf

Toro, G. V. R., Neves, A. S., \& Rezende, P. C. M. (2010). Bullying, o exercício da violência no contexto escolar: reflexões sobre um sintoma social. Psicol Teor E Prática, 12 (1), 123-137. http://pepsic.bvsalud.org/scielo.php?script=sci_arttext\&pid=S1516-36872010000100011\&lng=pt\&tlng=pt.

Zequinão, M. A., Medeiros, P., Silva, J. L., Pereira, B. O., \& Cardoso, F. L. (2020). Sociometric Status of Participants Involved in School Bullying. Paidéia, 30, e3011. https://doi.org/10.1590/1982-4327e3011 